\title{
X-ray Polarimetry - a Tool for the Galactic center diagnosis
}

\section{Frédéric Marin*}

Astronomical Institute of the Academy of Sciences, Boční II 1401, CZ-14100 Prague, Czech Republic

E-mail: frederic.marineasu.cas.cz

Was the Milky Way galaxy a low-luminosity active galactic nucleus (AGN) in the past? Can we find traces of remnant structures supporting this idea? What is the three-dimensional arrangement of matter around our central supermassive black hole? A number of fundamental questions concerning our own Galactic center remain controversial. To reveal the structure of the high-energy sky around our galactic core, a technique more sensitive to the morphology of the emitters than spectroscopy is needed. In this lecture note, I describe how X-ray polarimetry can open a new observational window by precisely measuring the three-dimensional position of the scattering material in the Galactic center. The observed polarization degree and polarization position angle would also determine unambiguously the primary source of emission and trace the centennial history of our supermassive black hole by detecting echoes of its past activity thanks to astrophysical mirrors. Finally, the synergy between X-ray polarimetry and infrared and radio observations can be used to better constrain the geometry in the first hundred parsecs around the Galactic center.

XI Multifrequency Behaviour of High Energy Cosmic Sources Workshop

25-30 May 2015

Palermo, Italy

${ }^{*}$ Speaker. 


\section{The Galactic center region}

At a distance of $8.33 \pm 0.35 \mathrm{kpc}$ [9] from our Solar system is an astrophysical environment that presents an extremely rich collection of gaseous, stellar and gravitational components: the Galactic center (GC). Due to the GC proximity, the angular size of its central supermassive black hole (9.65 $\mu$ as) may become resolvable in the near future thanks to very-long-baseline interferometry in the radio domain, making the Milky Way an excellent laboratory to locally investigate the processes happening in more distant galaxies. Until then, observations are studying Galactic regions up to a few hundred parsecs from the GC.

Within this Galactic volume, the stellar population is rich in young and massive stars, which is characteristic of a recent and/or ongoing star formation. The stars are essentially concentrated in a disk-like structure called the nuclear stellar disk, extending up to $230 \mathrm{pc}$ in width and $45 \mathrm{pc}$ in height; with stellar clusters being more frequent in the center of the nuclear bulge [10]. At similar distance scales, the maximum concentration of interstellar gas is gathered in an inclined molecular and atomic disk that extends up to $1.5 \mathrm{kpc}$ from the center, carrying about $10^{7} \mathrm{M}_{\odot}$ of $\mathrm{H} \mathrm{I}$ [5]. This disk reaches the inner $2 \mathrm{pc}$ of the GC, where it forms a non axisymmetric reservoir of gas with total mass of $2-5 \times 10^{5} \mathrm{M}_{\odot}$ [16]. This dense, warm, molecular and atomic region is seen nearly edge-on $\left(\leq 20^{\circ}\right)$, extends up to $3 \mathrm{pc}$ along the North-East direction and up to $7 \mathrm{pc}$ along the South-West [8], and is usually denoted as the circumnuclear disk (CND). More generally, the first hundred parsecs around the CND is rich in molecular gas, so that this region is named the central molecular zone (CMZ). The central two parsecs of the GC are occupied by the Sgr A West region which appears to be dominated by ionized gas emission. Finally, at the very center is a radio-intense, sub-arcsecond, structure that has been discovered in 1974 [2] and later associated with a strong gravitational field that governs the orbit of nearby stars [7]. This is considered as the first reliable identification of a supermassive black hole.

The GC is thus a vast concentration of molecular and atomic gaseous regions, bright star clusters, dynamical structures and, more importantly, a central supermassive black hole ( $\operatorname{Sgr} \mathrm{A}^{*}$ ). Its proximity allows us to probe the direct environment of a $>10^{5} \mathrm{M}_{\odot}$ compact region and better constrain the growth mechanism of black holes, the physical condition of the early Universe and the formation of galaxies.

\section{X-ray polarimetry as a new observational window}

Despite its proximity and the numerous radio to $\gamma$-ray observations of the GC, the history of the central parsecs of the Milky Way remains questioned. The quiescence of Sgr $\mathrm{A}^{*}$, which accretes at a fairly slow rate $\left(10^{-8} \mathrm{M}_{\odot} \cdot \mathrm{y}^{-1}\right.$ near the event horizon [1] $)$, is in contradiction with past observations of nearby nebulae $[24,15]$. Situated at projected distances $<150 \mathrm{pc}$, a collection of molecular gas clouds has shown to have "strong" $\left(L_{X} \sim 10^{35} \operatorname{ergs~s}^{-1}\right.$ [15]) absorption-corrected $\mathrm{X}$-ray luminosities in comparison with $\mathrm{Sgr} \mathrm{A}^{*}\left(\sim 2.4 \times 10^{33} \mathrm{ergs} \mathrm{s}^{-1}\right.$ [1]). Their spectra can be described by a power-law continuum and a prominent $6.4 \mathrm{keV}$ iron emission line, both being attributed to Compton scattering and fluorescence processes. The hypothesis of an irradiating Xray source located inside the nebulae has been ruled out by the absence of significant long-term variability of the iron $\mathrm{K} \alpha$ line [18]. In addition, the observed disagreement between the molecular 


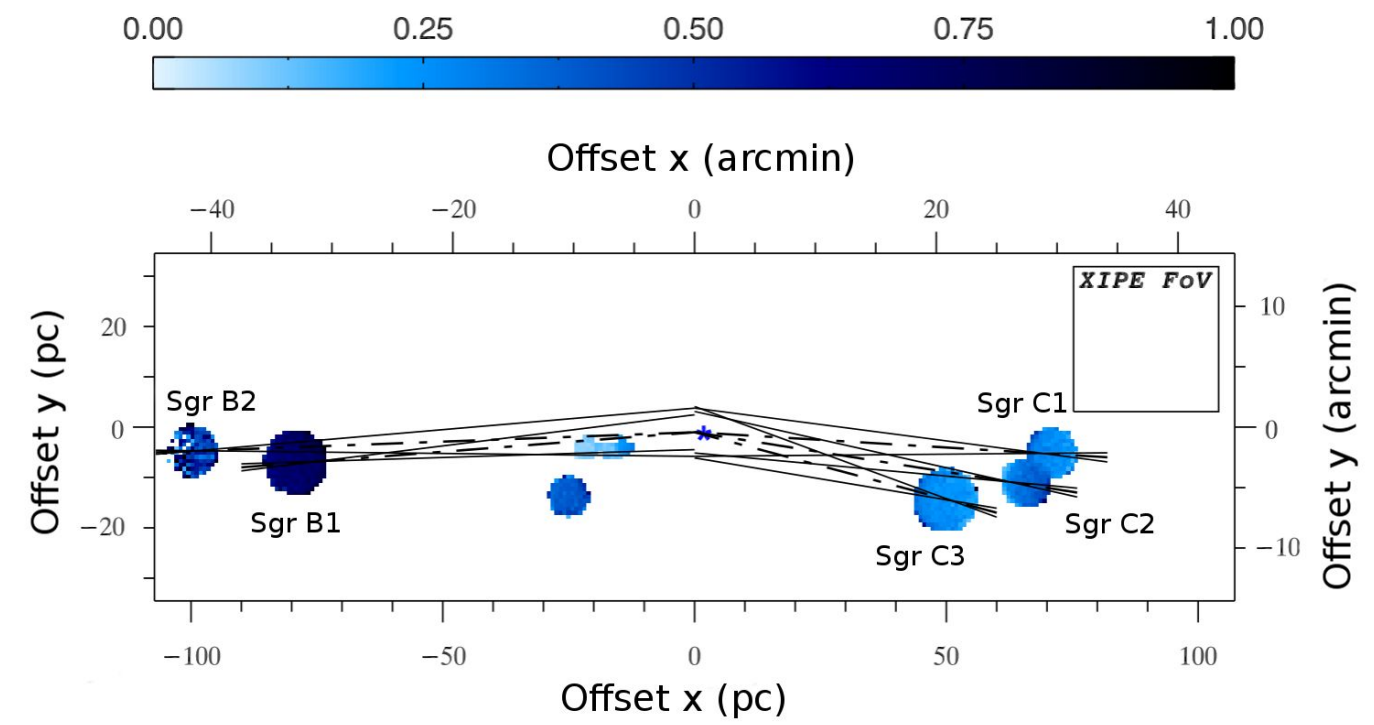

Figure 1: Simulated $4-8 \mathrm{keV}$ polarimetric map of the GC, using the point spread function of the XIPE mission. The polarization degree is color-coded and ranges from white $(0 \%)$ to blue $(100 \%)$. Eleven of the most observed reflection nebulae has been simulated to observe the effect of scattering-induced linear polarization. Dark segments are representative of the estimated polarization position angle (dashed line) and its associated error (solid line). The field of view (FoV) of XIPE is indicated with a white box, and a blue star indicates the position of Sgr A*.

mass distribution of the Sgr B2 cloud and the location of the peak of the extended $6.4 \mathrm{keV}$ emission suggests an external source that would be located in the vicinity of $\operatorname{Sgr~}^{*}$ [15]. One possible conclusion is that the $6.4 \mathrm{keV}$ bright, giant molecular clouds are reprocessing a past $\mathrm{X}$-ray activity of $\mathrm{Sgr} \mathrm{A}^{*}$, of which the duration and intensity are debated.

To evaluate the intensity of the activity period (not to be mistaken with short-lived X-ray flares nowadays observed in the GC, e.g. [17]), one can relate the intrinsic luminosity of the source to the iron $\mathrm{K} \alpha$ line flux and to the inverse of the distance to Sgr $\mathrm{A}^{*}$. If the former is known (with typical uncertainties lower than $15 \%$ ), the latter is restricted to the observed projected distance between the supermassive black hole and the reflection nebula. X-ray spectroscopy [20] and radio observations [21] were used to infer the position of the scattering nebulae but their results suffer from large uncertainties that does not allow to precisely constraint the solid angle of the cloud from the location of the primary source. This is a crucial piece of information (together with the cloud optical depth) to properly constrain the history and morphology of the GC.

Information about the spatial location of the last scattering event are encoded in photons by a phenomenon called polarization. In white light, most sources of light are incoherent and randomly polarized (over time the polarization is constantly changing in an unpredictable manner), but scattering can force the electric field vector to oscillate in a given plane, resulting in measurable polarization. Reflection nebulae are thus potential targets for X-ray polarimetry. Moreover, the degree of scatter-induced polarization is strongly correlated with the angle between the source, the mirror and the observer. Based on these properties, it has been proved that the molecular cloud Sgr B2 should produce large $(>30 \%)$ degrees of linear polarization in the $2-8 \mathrm{keV} \mathrm{[6]} \mathrm{and} 8$ 


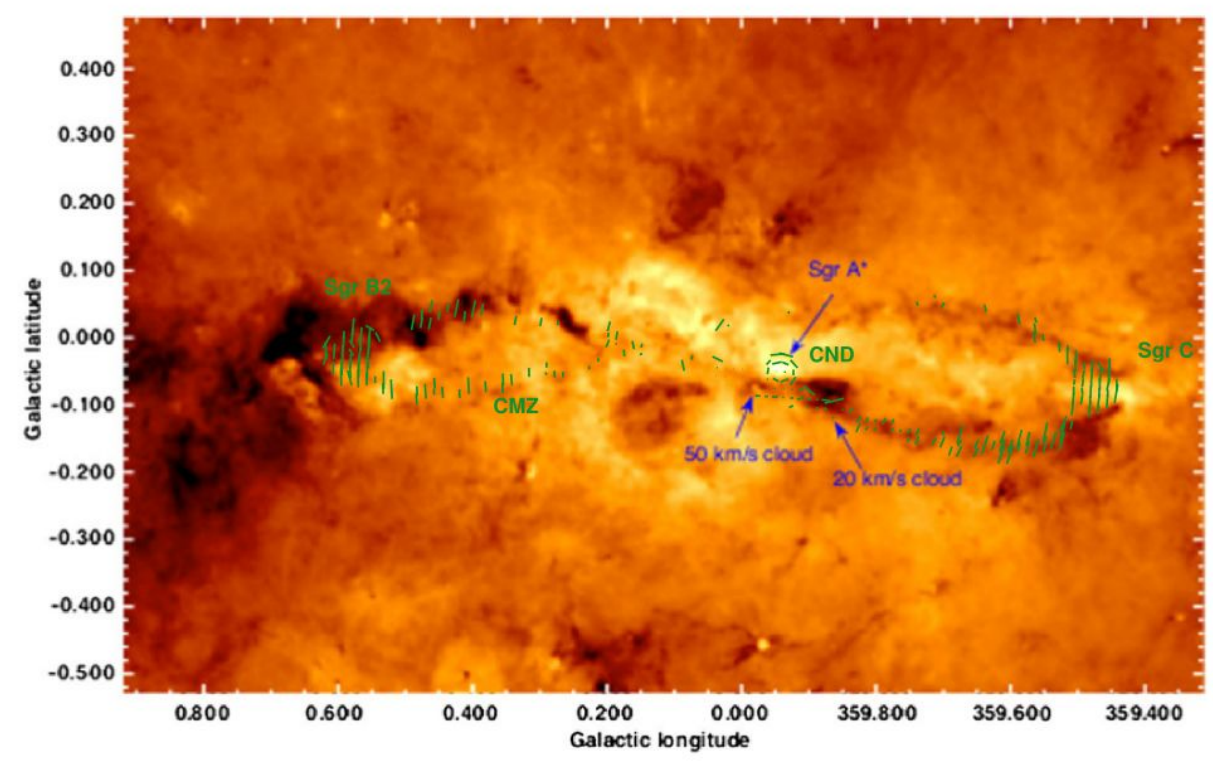

Figure 2: An example of broadband synergy between IR spectroscopy (temperature map from [14]) and $\mathrm{X}$-ray polarimetry (green lines, representative of the polarization degree and polarization position angle [11]) in the Galactic center. The central, quiescent, SMBH Sgr A* is revealed by its imprints onto the central molecular zone (CMZ), its circumnuclear disc (CND) and on the reflection nebulae (Sgr B and C complexes).

- $35 \mathrm{keV}[11,12]$ regimes, if the primary source of emission is Sgr $\mathrm{A}^{*}$. Using more advanced simulations, a $4-8 \mathrm{keV}$ polarization map of the $100 \times 30 \mathrm{pc}$ around $\mathrm{Sgr}^{*}$ has been produced (see Fig. 1 and [13]). The resulting polarization of the eleven most observed/constrained reflection nebulae (Sgr B1, Sgr B2, G0.11-0.11, Bridge E, Bridge D, Bridge B2, MC2, MC1, Sgr C3, $\mathrm{Sgr} \mathrm{C}$, and $\mathrm{Sgr} \mathrm{C} 1$ ) presents a variety of signatures, ranging from nearly unpolarized (The Bridge) to highly polarized ( $\sim 77 \%$ for the Sgr B complex) fluxes, with their polarization position angle normal to the scattering plane. Despite strong dilution due to a diffuse plasma emission ${ }^{1}$ angularly superimposed to the X-ray emission of the reflection nebulae, the Sgr B and Sgr C complexes, and the G0.11-0.11 cloud, have proven to be in the detectability range of a modern X-ray polarimeter. The X-ray Imaging Polarimetry Explorer (XIPE [23]), a mission concept selected as a candidate for the ESA's next medium-class science mission, is a prototypical satellite that could detect the polarization of these reflection nebulae. With a 3 Ms long observation of the Sgr B and C complexes, both the degree of polarization (related to the three-dimensional position of the cloud) and the polarization position angles (pinpointing the angular position of the illuminating source) would be detectable [13].

\section{A multi-wavelength complementarity}

Thanks to X-ray polarimetry, the spatial location of the reflection nebulae with respect to the primary source can be deduced. Additionally, measuring the polarization position angle would un-

\footnotetext{
${ }^{1}$ This two-temperature plasma is probably due to a multitude of faint sources (accreting white dwarfs and coronally active stars [19]).
} 
ambiguously point towards the primary source (see Fig. 1), giving proof or rejecting the hypothesis that the GC was active in the past (with an activity similar to a low-luminosity AGN).

Traces of this past activity are difficult to assess at other wavebands. One of the most promising hints is a continuous chain of irregular clumps around the Galactic core that has been revealed by the far-infrared cameras on-board of Herschel [14]. This elliptical twisted ring of molecular material is part of the $\mathrm{CMZ}$, and extends up to $\sim 100 \mathrm{pc}$ in longitude and $\sim 25 \mathrm{pc}$ in latitude. The coherence of the ring's morphology indicates stability over time and it is reminiscent of the dusty tori surrounding the central regions of AGN. A LMT/AzTEC $1.1 \mathrm{~mm}$ survey of the CMZ (D. Wang, private communication) confirms the existence of this peculiar structure. Completely optically thin to dust emission, the conjugation of this radio map with maps at shorter wavelengths will allow to put strong dust-based constraints on the mass of dense clouds. Together with multi-band nearinfrared images, it might be possible to determine the line-of-sight locations of the clouds based on extinction measurements. Until then, the geometrical size of the CMZ, its column density in excess of $10^{24} \mathrm{~cm}^{-2}$, and its orbital speed $\left(\sim 100 \mathrm{~km} \mathrm{~s}^{-1}\right)$, are all compatible with AGN tori [22].

As an illustration of the power of X-ray polarimetry in combination with other wavelengths, results from $8-35 \mathrm{keV}$ polarimetric simulations of the GC [11], including the Sgr B and C complexes, the CND, and the $\infty$-shaped CMZ, have been superimposed on the temperature map from [14] in Fig. 2. The CMZ polarization follows the morphology of the twisted structure, with partial disappearance of the polarization vectors due to low polarization degrees induced by unfavorable scattering geometries and dilution from the unpolarized primary flux. Maximum polarization is expected at the Eastern and Western quadratures, where the CMZ mixes with the giant molecular clouds.

\section{Acknowledgments}

I would like to acknowledge my collaborators Fabio Muleri, Paollo Soffitta, Vladimír Karas and Devaky Kunneriath for the work accomplished together in the topic of X-ray polarimetry. I am also grateful to Sergio Molinari who kindly accepted to share his 2011's temperature map of the Galactic center region, Michal Švanda who helped me to create the IR/X-ray map, and to Daniel Wang for his insights on the radio domain.

\section{References}

[1] Baganoff, F. K., Maeda, Y., Morris, M., et al., Chandra X-Ray Spectroscopic Imaging of Sagittarius $A^{*}$ and the Central Parsec of the Galaxy, ApJ 591 (891) [arXiv:astro-ph/0102151].

[2] Balick, B., \& Brown, R. L., Intense sub-arcsecond structure in the galactic center, ApJ 194 (265).

[3] Bender, R., Kormendy, J., Bower, G., et al., HST STIS Spectroscopy of the Triple Nucleus of M31: Two Nested Disks in Keplerian Rotation around a Supermassive Black Hole, ApJ 631 (280) [arXiv:astro-ph/0509839].

[4] Bettoni, D., Falomo, R., Fasano, G., \& Govoni, F., The black hole mass of low redshift radiogalaxies, $A \& A 399$ (869) [arXiv:astro-ph/0212162].

[5] Burton, W. B., \& Liszt, H. S., The gas distribution in the central region of the Galaxy. I - Atomic hydrogen, ApJ 225 (815). 
[6] Churazov, E., Sunyaev, R., \& Sazonov, S., Polarization of X-ray emission from the Sgr B2 cloud, MNRAS 330 (817) [arXiv: astro-ph/ 0111065 ].

[7] Eckart, A., Genzel, R., Ott, T., \& Schödel, R., Stellar orbits near Sagittarius A*, MNRAS 331 (917) [arXiv:astro-ph/0201031].

[8] Genzel, R., Crawford, M. K., Townes, C. H., \& Watson, D. M., The neutral-gas disk around the galactic center, ApJ 297 (766).

[9] Gillessen, S., Eisenhauer, F., Trippe, S., et al., Monitoring Stellar Orbits Around the Massive Black Hole in the Galactic Center, ApJ 692 (1075) [arXiv: 0810 . 4674 ].

[10] Launhardt, R., Zylka, R., \& Mezger, P. G., The nuclear bulge of the Galaxy. III. Large-scale physical characteristics of stars and interstellar matter, A\&A 384 (112) [arXiv: astro-ph/0201294].

[11] Marin, F., Karas, V., Kunneriath, D., \& Muleri, F., Prospects of 3D mapping of the Galactic Centre clouds with X-ray polarimetry, MNRAS 441 (3170) [arXiv: 1405 . 0898].

[12] Marin, F., Karas, V., Kunneriath, D., Muleri, F., \& Soffitta, P., Probing the Galactic center with X-ray polarimetry, Proc. of the SF2A 109 [arXiv: 1408.0354 ].

[13] Marin, F., Muleri, F., Soffitta, P., Karas, V., \& Kunneriath, D., Reflection nebulae in the Galactic center: soft X-ray imaging polarimetry, A\&A 576 (A19) [arXiv: 1502 . 04894 ].

[14] Molinari, S., Bally, J., Noriega-Crespo, A., et al., A 100 pc Elliptical and Twisted Ring of Cold and Dense Molecular Clouds Revealed by Herschel Around the Galactic Center, ApJL 735 (L33) [arXiv:1105.5486].

[15] Murakami, H., Koyama, K., Sakano, M., Tsujimoto, M., \& Maeda, Y., ASCA Observations of the Sagittarius B2 Cloud: An X-Ray Reflection Nebula, ApJ 534 (283) [arXiv: astro-ph/9908229].

[16] Oka, T., Nagai, M., Kamegai, K., \& Tanaka, K., A New Look at the Galactic Circumnuclear Disk, ApJ 732 (120).

[17] Porquet, D., Predehl, P., Aschenbach, B., et al., XMM-Newton observation of the brightest X-ray flare detected so far from Sgr A*, A\&A 407 (L17) [arXiv: astro-ph/0307110].

[18] Revnivtsev, M. G., Churazov, E. M., Sazonov, S. Y., et al., Hard X-ray view of the past activity of Sgr $A^{*}$ in a natural Compton mirror, A\&A 425 (L49) [arXiv: astro-ph/ 0408190 ].

[19] Revnivtsev, M., Vikhlinin, A., \& Sazonov, S., Resolving the Galactic X-ray background, A\&A 473 (857) [arXiv:astro-ph/0611952].

[20] Ryu, S. G., Koyama, K., Nobukawa, M., Fukuoka, R., \& Tsuru, T. G., An X-Ray Face-on View of the Sgr B Molecular Clouds Observed with Suzaku, PASJ 61 (751) [arXiv: 0904 . 4550].

[21] Sawada, T., Hasegawa, T., Handa, T., \& Cohen, R. J., A Molecular Face-on View of the Galactic Centre Region, MNRAS 349 (1167) [arXiv: astro-ph/ 0401286 ].

[22] Shi, Y., Rieke, G. H., Hines, D. C., et al., $9.7 \mu \mathrm{m}$ Silicate Features in Active Galactic Nuclei: New Insights into Unification Models, ApJ 653 (127) [arXiv: astro-ph/ 0608645 ].

[23] Soffitta, P., Barcons, X., Bellazzini, R., et al., XIPE: the X-ray imaging polarimetry explorer, Experimental Astronomy 36 (523) [arXiv: 1309.6995$].$

[24] Sunyaev, R. A., Markevitch, M., \& Pavlinsky, M., The center of the Galaxy in the recent past - A view from GRANAT, ApJ $\mathbf{4 0 7}$ (606). 